UDC 517.956

\title{
HOMOGENIZATION OF THE ROBIN PROBLEM IN A THICK MULTILEVEL JUNCTION
}

\section{УСЕРЕДНЕННЯ ЗАДАЧІ РОБІНА В ГУСТОМУ БАГАТОРІВНЕВОМУ З'СДНАННІ}

\section{U. De Maio}

Università degli Studi di Napoli Federico II

Complesso Monte S. Angelo-Edificio "T", Via Cintia, 80126 Napoli, Italia

e-mail: udemaio@unina.it

\section{T. A. Mel'nyk}

Kyiv Nat. Taras Shevchenko Univ.

Volodymyrs'ka Str. 64, 01033, Kyiv, Ukraine

e-mail:melnyk@imath.kiev.ua

\section{Perugia}

Università degli Studi di Napoli Federico II

Complesso Monte S. Angelo-Edificio "T", Via Cintia, 80126 Napoli, Italia

e-mail:perugia@unina.it

In the paper we consider a mixed boundary-value problem for the Poisson equation in a plane two-level junction $\Omega_{\varepsilon}$, which is the union of a domain $\Omega_{0}$ and a large number $2 N$ of thin rods with variable thickness of order $\varepsilon=\mathcal{O}\left(N^{-1}\right)$. The thin rods are divided into two levels depending on their length. In addition, the thin rods from each level are E-periodically alternated. We investigate the asymptotic behaviour of the solution as $\varepsilon \rightarrow 0$ under the Robin conditions on the boundaries of the thin rods. By using some special extension operators, the convergence theorem is proved.

Розглядається мішана крайова задача для рівняння Пуассона у плоскому дворівневому з'єднанні $\Omega_{\varepsilon}$, яке є об'єднанням деякої області $\Omega_{0}$ та великої кількості $2 N$ тонких стержнів із змінною товщиною порядку $\varepsilon=\mathcal{O}\left(N^{-1}\right)$. Тонкі стержні розділено на два рівні в залежності від їх довжини. Крім того, тонкі стержні з кожного рівня в-періодично чергуються. Вивчено асимптотичну поведінку розв'язку, коли $\varepsilon \rightarrow 0$, при крайових умовах Робіна на межах тонких стержнів. Із використанням спеціальних операторів продовження доведено теорему збіжності.

Introduction. In this paper we consider a new type of thick junctions, namely, thick multilevel junctions. A thick multilevel junction is the union of some domain, which is called the junction's body, and a large number $N=\mathcal{O}\left(\varepsilon^{-1}\right)$ of thin domains of thickness of order $\mathcal{O}(\varepsilon)$. Here $\varepsilon$ is a small parameter. The thin domains are divided into a finite number of levels depending on their length. In addition, the thin domains from each levels are $\varepsilon$-periodically alternated along some manifold on the boundary of the junction's body. This manifold is called the joint zone.

The aim of researches is to develop rigorous asymptotic methods for boundary-value problems in thick multilevel junctions when the parameter $\varepsilon$ goes to 0 , i.e., when the number of the attached thin domains increases and their thickness decreases. The asymptotic methods,

(c) U. De Maio, T. A. Mel'nyk, and C. Perugia, 2004 
which were developed in [1-3] are used. A spectral problem in a plane thick multilevel junction with the flat boundaries of the thin rods was considered in [4]. Here we consider a mixed boundary-value problem for the Poisson equation in a plane thick two-level junction with variable thickness of the thin rods.

1. Statement of the problem. Let $a, d_{1}, d_{2}, b_{1}, b_{2}$ be positive real numbers and let $d_{1} \leq$ $\leq d_{2}, 0<b_{1}<b_{2}<1$. Consider two positive piecewise smooth functions $h_{1}$ and $h_{2}$ on the the segments $\left[-d_{1}, 0\right]$ and $\left[-d_{2}, 0\right]$, respectively. Suppose the functions $h_{1}$ and $h_{2}$ satisfy the following conditions:

$$
\begin{gathered}
\exists \delta_{0} \in\left(b_{1}, b_{2}\right) \forall x_{2} \in\left[-d_{1}, 0\right]: 0<b_{1}-h_{1}\left(x_{2}\right) / 2, \quad b_{1}+h_{1}\left(x_{2}\right) / 2<\delta_{0} ; \\
\forall x_{2} \in\left[-d_{2}, 0\right]: \delta_{0}<b_{2}-h_{2}\left(x_{2}\right) / 2, \quad b_{2}+h_{2}\left(x_{2}\right) / 2<1 .
\end{gathered}
$$

It follows from these assumptions that there exist positive constants $m_{0}, M_{0}$ such that

$$
\begin{gathered}
0<m_{0} \leq h_{1}\left(x_{2}\right)<\delta_{0} \quad \text { and } \quad\left|h_{1}^{\prime}\left(x_{2}\right)\right| \leq M_{0} \quad \text { a. e. in }\left[-d_{1}, 0\right], \\
0<m_{0} \leq h_{2}\left(x_{2}\right)<1-\delta_{0} \quad \text { and } \quad\left|h_{2}^{\prime}\left(x_{2}\right)\right| \leq M_{0} \quad \text { a. e. in }\left[-d_{2}, 0\right]
\end{gathered}
$$

Let us divide segment $[0, a]$ into $N$ equal segments $[\varepsilon j, \varepsilon(j+1)], j=0, \ldots, N-1$. Here $N$ is a large integer, therefore, the value $\varepsilon=a / N$ is a small discrete parameter.

A model plane thick two-level junction $\Omega_{\varepsilon}$ consists of the junction's body

$$
\Omega_{0}=\left\{x \in \mathbb{R}^{2}: 0<x_{1}<a, \quad 0<x_{2}<\gamma\left(x_{1}\right)\right\},
$$

where $\gamma \in C^{1}([0, a]), \gamma(0)=\gamma(a), \min _{[0, a]} \gamma>0$, and a large number of the thin rods

$$
\begin{aligned}
& G_{j}^{(1)}(\varepsilon)=\left\{x \in \mathbb{R}^{2}:\left|x_{1}-\varepsilon\left(j+b_{1}\right)\right|<\varepsilon h_{1}\left(x_{2}\right) / 2, \quad x_{2} \in\left(-d_{1}, 0\right]\right\}, \quad j=0,1, \ldots, N-1, \\
& G_{j}^{(2)}(\varepsilon)=\left\{x \in \mathbb{R}^{2}:\left|x_{1}-\varepsilon\left(j+b_{2}\right)\right|<\varepsilon h_{2}\left(x_{2}\right) / 2, \quad x_{2} \in\left(-d_{2}, 0\right]\right\}, \quad j=0,1, \ldots, N-1,
\end{aligned}
$$

i.e.,

$$
\Omega_{\varepsilon}=\Omega_{0} \cup G^{(1)}(\varepsilon) \cup G^{(2)}(\varepsilon),
$$

where $G^{(1)}(\varepsilon)=\cup_{j=0}^{N-1} G_{j}^{(1)}(\varepsilon), G^{(2)}(\varepsilon)=\cup_{j=0}^{N-1} G_{j}^{(2)}(\varepsilon)$.

We see that the number of the thin rods is equal to $2 N$ and they are divided into two levels $G^{(1)}(\varepsilon)$ and $G^{(2)}(\varepsilon)$ depending on their length (we recall that $d_{1} \leq d_{2}$ ). The small parameter $\varepsilon$ characterizes the distance between the thin neighboring rods and their thickness. The thickness of the rods from the first level is equal to $\varepsilon h_{1}$ and to $\varepsilon h_{2}$ for the rods from the second level. These thin rods from each level are $\varepsilon$-periodically alternated along the segment $I_{0}=\left\{x: x_{1} \in\right.$ $\left.\in[0, a], x_{2}=0\right\}$.

Denote by $\Upsilon_{j}^{(i, \pm)}(\varepsilon)$ the lateral surfaces of the thin $\operatorname{rod} G_{j}^{(i)}(\varepsilon)$; the signs " + "or " - " indicate the right or left surface respectively. The base of $G_{j}^{(i)}(\varepsilon)$ will be denoted by $\Theta_{j}^{(i)}(\varepsilon)$. Also we introduce the following notations:

$$
\Upsilon^{(i, \pm)}(\varepsilon):=\bigcup_{j=0}^{N-1} \Upsilon_{j}^{(i, \pm)}(\varepsilon), \quad \Theta^{(i)}(\varepsilon):=\bigcup_{j=0}^{N-1} \Theta_{j}^{(i)}(\varepsilon)
$$




$$
\Upsilon^{(i)}(\varepsilon):=\Upsilon^{(i,+)}(\varepsilon) \cup \Upsilon^{(i,-)}(\varepsilon) \cup \Theta^{(i)}(\varepsilon)
$$

for $i=1,2$.

In $\Omega_{\varepsilon}$ we consider the following mixed boundary-value problem:

$$
\begin{aligned}
-\Delta_{x} u_{\varepsilon}(x) & =f_{\varepsilon}(x), & & x \in \Omega_{\varepsilon}, \\
\partial_{\nu} u_{\varepsilon}(x) & =-\varepsilon k_{1} u_{\varepsilon}(x), & & x \in \Upsilon^{(1)}(\varepsilon), \\
\partial_{\nu} u_{\varepsilon}(x) & =-\varepsilon k_{2} u_{\varepsilon}(x), & & x \in \Upsilon^{(2)}(\varepsilon), \\
\partial_{x_{1}}^{p} u_{\varepsilon}\left(0, x_{2}\right) & =\partial_{x_{1}}^{p} u_{\varepsilon}\left(a, x_{2}\right), & & x_{2} \in[0, \gamma(0)], p=0,1, \\
\partial_{\nu} u_{\varepsilon}(x) & =0, & & x \in \Gamma_{\varepsilon} .
\end{aligned}
$$

Here $\partial_{\nu}=\partial / \partial \nu$ is the outward normal derivative, $\partial_{x_{1}}=\partial / \partial x_{1}$, the constants $k_{1}$ and $k_{2}$ are positive. Thus, we have the Robin conditions on the boundaries of the thin rods, the periodic conditions on the vertical sides of $\Omega_{0}$ and the Neumann condition on the other part $\Gamma_{\varepsilon}$ of $\partial \Omega_{\varepsilon}$.

We can regard without loss of generality that the right-hand side $f_{\varepsilon}$ belongs to $L^{2}\left(\Omega_{2}\right)$, where $\Omega_{2}$ is the interior of $\overline{\Omega_{0}} \cup \overline{D_{2}}, D_{2}=(0, a) \times\left(-d_{2}, 0\right)$ is a rectangle that is filled up by the thin rods from the second level in the limit passage as $\varepsilon \rightarrow 0$. Similarly, $D_{1}=(0, a) \times\left(-d_{1}, 0\right)$ and $\Omega_{1}$ is the interior of $\overline{\Omega_{0}} \cup \overline{D_{1}}$.

We assume that

$$
f_{\varepsilon} \rightarrow f_{0} \quad \text { in } \quad L^{2}\left(\Omega_{2}\right) \quad \text { as } \quad \varepsilon \rightarrow 0
$$

The aim of our research is to study the asymptotic behaviour of the solution to problem (2) as $\varepsilon \rightarrow 0$, i.e., when the number of attached thin rods infinitely increases and their thickness tends to 0 .

2. Auxiliary inequalities. First we recall that for every fixed value $\varepsilon$, in accordance with the main results of the theory of boundary-value problems, there exists a unique weak solution $u_{\varepsilon} \in \mathcal{H}_{\varepsilon}$ to problem (2) such that the integral identity

$$
\int_{\Omega_{\varepsilon}} \nabla u_{\varepsilon} \cdot \nabla \varphi d x+\varepsilon k_{1} \int_{\Upsilon^{(1)}(\varepsilon)} u_{\varepsilon} \varphi d l_{x}+\varepsilon k_{2} \int_{\Upsilon^{(2)}(\varepsilon)} u_{\varepsilon} \varphi d l_{x}=\int_{\Omega_{\varepsilon}} f_{\varepsilon}(x) \varphi(x) d x
$$

holds for any function $\varphi \in \mathcal{H}_{\varepsilon}$, where

$$
\mathcal{H}_{\varepsilon}=\left\{u \in H^{1}\left(\Omega_{\varepsilon}\right): \partial_{x_{1}}^{p} u\left(0, x_{2}\right)=\partial_{x_{1}}^{p} u\left(a, x_{2}\right), x_{2} \in[0, \gamma(0)], \quad p=0,1\right\}
$$

In addition, the solution $u_{\varepsilon}$ satisfies the inequality

$$
\left\|u_{\varepsilon}\right\|_{H^{1}\left(\Omega_{\varepsilon}\right)} \leq c_{1}\left\|f_{\varepsilon}\right\|_{L^{2}\left(\Omega_{\varepsilon}\right)} .
$$

Let us show that the constant $c_{1}$ in (5) is independent of the small parameter $\varepsilon$. 
Lemma 1. For $\varepsilon$ small enough, the usual norm $\|\cdot\|_{H^{1}\left(\Omega_{\varepsilon}\right)}$ in the Sobolev space $H^{1}\left(\Omega_{\varepsilon}\right)$ and the norm:

$$
\|v\|_{\varepsilon, k_{1}, k_{2}}=\left(\int_{\Omega_{\varepsilon}}|\nabla v|^{2} d x+\varepsilon k_{1} \int_{\Upsilon^{(1)}(\varepsilon)} v^{2} d l_{x}+\varepsilon k_{2} \int_{\Upsilon^{(2)}(\varepsilon)} v^{2} d l_{x}\right)^{1 / 2}
$$

are uniformly equivalent, i.e., there exist constants $C_{1}>0, C_{2}>0$ and $\varepsilon_{0}$ such that for all $\varepsilon \in\left(0, \varepsilon_{0}\right)$ and any function $v \in H^{1}\left(\Omega_{\varepsilon}\right)$ the inequalities

$$
C_{1}\|v\|_{H^{1}\left(\Omega_{\varepsilon}\right)} \leq\|v\|_{\varepsilon, k_{1}, k_{2}} \leq C_{2}\|v\|_{H^{1}\left(\Omega_{\varepsilon}\right)}
$$

are satisfied.

Proof. Let us defined the following function:

$$
Y(t)= \begin{cases}-t+b_{1}, & t \in\left[0, \delta_{0}\right) \\ -t+b_{2}, & t \in\left[\delta_{0}, 1\right)\end{cases}
$$

and then periodically extend it to $\mathbb{R}$.

Integrating by parts the integral $\varepsilon \int_{G^{(1)}(\varepsilon) \cup G^{(2)}(\varepsilon)} Y\left(x_{1} / \varepsilon\right) \partial_{x_{1}} v d x$ and taking into account that the outward normal to the lateral surfaces $\Upsilon_{j}^{(i, \pm)}(\varepsilon)$ of the thin $\operatorname{rod} G_{j}^{(i)}(\varepsilon)$, except for some set of measure zero, has the form

$$
\nu_{ \pm}^{(i)}(\varepsilon)=\frac{1}{\sqrt{1+\varepsilon^{2} 4^{-1}\left|h_{i}^{\prime}\left(x_{2}\right)\right|^{2}}}\left( \pm 1,-\varepsilon \frac{h_{i}^{\prime}\left(x_{2}\right)}{2}\right), \quad i=1,2, \quad j=0, \ldots, N-1
$$

we get the identity

$$
\begin{aligned}
\varepsilon \sum_{i=1}^{2} \int_{\Upsilon(i, \pm)} \frac{h_{i}\left(x_{2}\right)}{2 \sqrt{1+\varepsilon^{2} 4^{-1}\left|h_{i}^{\prime}\left(x_{2}\right)\right|^{2}}} v d l_{x}= & \\
& =\int_{G^{(1)}(\varepsilon) \cup G^{(2)}(\varepsilon)} v d x-\varepsilon \int_{G^{(1)}(\varepsilon) \cup G^{(2)}(\varepsilon)} Y\left(\frac{x_{1}}{\varepsilon}\right) \partial_{x_{1}} v d x \quad \forall v \in H^{1}\left(\Omega_{\varepsilon}\right) .
\end{aligned}
$$

Using the identity (9), the properties of the trace operator and taking into account that $\max _{\mathbb{R}}|Y| \leq 1$, we obtain 


$$
\begin{aligned}
& \|v\|_{\varepsilon, k_{1}, k_{2}}^{2}=\int_{\Omega_{\varepsilon}}|\nabla v|^{2} d x+ \\
& +\varepsilon \sum_{i=1}^{2} k_{i} \int_{\Upsilon_{(i, \pm)}(\varepsilon)} \frac{2 \sqrt{1+\varepsilon^{2} 4^{-1}\left|h_{i}^{\prime}\left(x_{2}\right)\right|^{2}}}{h_{i}\left(x_{2}\right)} \frac{h_{i}\left(x_{2}\right)}{2 \sqrt{1+\varepsilon^{2} 4^{-1}\left|h_{i}^{\prime}\left(x_{2}\right)\right|^{2}}} v^{2} d l_{x}+ \\
& +\varepsilon \sum_{i=1}^{2} k_{i} \int_{\Theta^{(i)}(\varepsilon)} v^{2} d x_{1} \leq \\
& \leq \int_{\Omega_{\varepsilon}}|\nabla v|^{2} d x+c_{1} \sum_{i=1}^{2} \int_{\Upsilon(i, \pm)(\varepsilon)} \frac{\varepsilon h_{i}\left(x_{2}\right)}{2 \sqrt{1+\varepsilon^{2} 4^{-1}\left|h_{0}^{\prime}\left(x_{2}\right)\right|^{2}}} v^{2} d l_{x}+\varepsilon c_{2} \sum_{i=1}^{2}\|v\|_{H^{1}\left(G^{(i)}(\varepsilon)\right)}^{2} \leq \\
& \leq c_{3}\|v\|_{H^{1}\left(\Omega_{\varepsilon}\right)}^{2}+c_{1}\left(\int_{G^{(1)}(\varepsilon) \cup G^{(2)}(\varepsilon)} v^{2} d x-\varepsilon \int_{G^{(1)}(\varepsilon) \cup G^{(2)}(\varepsilon)} Y\left(\frac{x_{1}}{\varepsilon}\right) 2 v \partial_{x_{1}} v d x\right) \leq \\
& \leq c_{3}\|v\|_{H^{1}\left(\Omega_{\varepsilon}\right)}^{2}+c_{1}\left(\int_{G^{(1)}(\varepsilon) \cup G^{(2)}(\varepsilon)} v^{2} d x+\int_{G^{(1)}(\varepsilon) \cup G^{(2)}(\varepsilon)} \varepsilon\left(\left(\partial_{x_{1}} v\right)^{2}+v^{2}\right) d x\right) \leq \\
& \leq C_{2}\|u\|_{H^{1}\left(\Omega_{\varepsilon}\right)}^{2} .
\end{aligned}
$$

Similarly, we obtain

$$
\begin{aligned}
& \|v\|_{H^{1}\left(\Omega_{\varepsilon}\right)}^{2}=\int_{\Omega_{\varepsilon}}|\nabla v|^{2} d x+\int_{\Omega_{0}} v^{2} d x+\int_{G^{(1)}(\varepsilon) \cup G^{(2)}(\varepsilon)} v^{2} d x=\int_{\Omega_{\varepsilon}}|\nabla v|^{2} d x+\int_{\Omega_{0}} v^{2} d x+ \\
& \quad+\varepsilon \sum_{i=1}^{2} \int_{\Upsilon^{(i, \pm)}(\varepsilon)} \frac{h_{i}\left(x_{2}\right)}{2 \sqrt{1+\varepsilon^{2} 4^{-1}\left|h_{i}^{\prime}\left(x_{2}\right)\right|^{2}}} v^{2} d l_{x}+\varepsilon \int_{G^{(1)}(\varepsilon) \cup G^{(2)}(\varepsilon)} Y\left(\frac{x_{1}}{\varepsilon}\right) 2 v \partial_{x_{1}} v d x \leq \\
& \leq C_{3}\|v\|_{\varepsilon, k_{1}, k_{2}}^{2}+\int_{\Omega_{0}} v^{2} d x+\varepsilon \int_{G^{(1)}(\varepsilon) \cup G^{(2)}(\varepsilon)} v^{2} d x,
\end{aligned}
$$

whence

$$
\|v\|_{H^{1}\left(\Omega_{\varepsilon}\right)}^{2} \leq C_{4}\left(\|v\|_{\varepsilon, k_{1}, k_{2}}^{2}+\int_{\Omega_{0}} v^{2} d x\right) .
$$


Now let us show that there exists a positive constant $C_{5}$ such that for $\varepsilon$ small enough and for any $v \in H^{1}\left(\Omega_{\varepsilon}\right)$,

$$
\int_{\Omega_{0}} v^{2} d x \leq C_{5}\|v\|_{\varepsilon, k_{1}, k_{2}}^{2} .
$$

We argue by contradiction. If not, then there exist sequences $\left\{\varepsilon_{m}: m \in \mathbb{N}\right\}$ and $\left\{v_{m}\right\} \subset$ $\subset H^{1}\left(\Omega_{\varepsilon_{m}}\right)$ such that $\lim _{m \rightarrow 0} \varepsilon_{m}=0$,

$$
\begin{gathered}
\int_{\Omega_{0}} v_{m}^{2} d x=1 \\
\int_{\Omega_{\varepsilon_{m}}}\left|\nabla v_{m}\right|^{2} d x+\varepsilon_{m} \sum_{i=1}^{2} k_{i} \int_{\Upsilon^{(i)}\left(\varepsilon_{m}\right)} v_{m}^{2} d l_{x}<\frac{1}{m} .
\end{gathered}
$$

Since the sequence $\left\{v_{m}\right\}$ is bounded in $H^{1}\left(\Omega_{0}\right)$, we may assume without loss of generality that it is a Cauchy sequence in $L^{2}\left(\Omega_{0}\right)$. From inequality (14) it follows that $\left\{v_{m}\right\}$ is a Cauchy sequence also in $H^{1}\left(\Omega_{0}\right)$,

$$
\left\|v_{m}-v_{n}\right\|_{H^{1}\left(\Omega_{0}\right)}^{2} \leq\left\|v_{m}-v_{n}\right\|_{L_{2}\left(\Omega_{0}\right)}^{2}+\frac{1}{m}+\frac{1}{n} .
$$

Hence, $\left\{v_{m}\right\}$ converges to some element $v_{0} \in H^{1}\left(\Omega_{0}\right)$. Obviously, $v_{0} \equiv$ const in $H^{1}\left(\Omega_{0}\right)$ and, due to (13), $v_{0}=\left|\Omega_{0}\right|^{-1 / 2}$, where $\left|\Omega_{0}\right|$ denotes the measure of the domain $\Omega_{0}$.

Then, the sequence of the traces of $\left\{v_{m}\right\}$ converges to $v_{0}$ in $L^{2}\left(\partial \Omega_{0}\right)$ as well and it is easy to verify that

$$
\begin{aligned}
\int_{I_{0}\left(\varepsilon_{m}\right)} v_{m}^{2}\left(x_{1}, 0\right) d x_{1} & =\sum_{i=1}^{2} \int_{I_{0}} \chi_{i}\left(x_{1} / \varepsilon_{m}\right) v_{m}^{2}\left(x_{1}, 0\right) d x_{1} \rightarrow \\
& \rightarrow \sum_{i=1}^{2} h_{i}(0) \int_{I_{0}} v_{0}^{2}\left(x_{1}, 0\right) d x_{1}=\left(h_{1}(0)+h_{2}(0)\right)\left|\Omega_{0}\right|^{-1} a \neq 0, \quad m \rightarrow \infty,
\end{aligned}
$$

where $I_{0}(\varepsilon):=I_{0} \cap \Omega_{\varepsilon}$ and $\chi_{i}(\cdot)$ is a 1-periodic function such that

$$
\chi_{i}(t)= \begin{cases}1, & t \in\left(b_{i}-\frac{h_{i}(0)}{2}, b_{i}+\frac{h_{i}(0)}{2}\right), \\ 0, & t \in[0,1] \backslash\left(b_{i}-\frac{h_{i}(0)}{2}, b_{i}+\frac{h_{i}(0)}{2}\right),\end{cases}
$$


for $i=1,2$. Obviously,

$$
\chi_{i}\left(x_{1} / \varepsilon\right) \rightarrow \int_{0}^{1} \chi_{i}(t) d t=h_{i}(0) \quad \text { weakly in } L^{2}(0, a) \quad \text { as } \varepsilon \rightarrow 0 .
$$

On the other hand, from (9) and (14) it follows that

$$
\int_{G^{(1)}\left(\varepsilon_{m}\right) \cup G^{(2)}\left(\varepsilon_{m}\right)}\left(\left|\nabla v_{m}\right|^{2}+v_{m}^{2}\right) d x \leq \frac{C_{6}}{m}
$$

and, therefore,

$$
\int_{I_{0}\left(\varepsilon_{m}\right)} v_{m}^{2}\left(x_{1}, 0\right) d x_{1} \leq C_{7} \int_{\left.G^{(1)}\left(\varepsilon_{m}\right) \cup G^{(2)}\left(\varepsilon_{m}\right)\right)}\left(\left|\nabla v_{m}\right|^{2}+v_{m}^{2}\right) d x \leq \frac{C_{8}}{m},
$$

where the constants $C_{6}, C_{7}, C_{8}$ are independent of $m$. This means that

$$
\int_{I_{0}\left(\varepsilon_{m}\right)} v_{m}^{2}\left(x_{1}, 0\right) d x_{1} \rightarrow 0 \quad \text { as } m \rightarrow \infty .
$$

However (17) varies from (15). This contradiction establishes estimate (12).

Thus, by virtue of (11) and (12), we obtain the left inequality in (6).

The lemma is proved.

Remark 1. Hereafter all constants $\left\{c_{i}, C_{i}\right\}$ in asymptotic inequalities are independent of the parameter $\varepsilon$.

3. Extension operator. Due to the $a$-periodic condition in problem (2), we can assume that the function $f_{\varepsilon}$ and the solution $u_{\varepsilon}$ are $a$-periodic functions with respect to $x_{1}$.

Theorem 1. Let condition (3) be satisfied and, in addition, there exist constants $C_{1}$ and $\varepsilon_{0}$ such that for all values $\varepsilon \in\left(0, \varepsilon_{0}\right)$

$$
\int_{\Omega_{\varepsilon}}\left(F_{\varepsilon}(x)\right)^{2} d x \leq C_{1}
$$

where $F_{\varepsilon}(x)=\varepsilon^{-1}\left(f_{\varepsilon}\left(x+\varepsilon \bar{e}_{1}\right)-f_{\varepsilon}(x)\right) \quad\left(\bar{e}_{1}=(1,0)\right)$.

Then there exist extension operators

$$
\mathbf{P}_{\varepsilon}^{(1)}: H^{1}\left(\Omega_{0} \cup G^{(1)}(\varepsilon)\right) \mapsto H^{1}\left(\Omega_{1}\right) \text { and } \mathbf{P}_{\varepsilon}^{(2)}: H^{1}\left(\Omega_{0} \cup G^{(2)}(\varepsilon)\right) \mapsto H^{1}\left(\Omega_{2}\right)
$$

such that, for the solution $u_{\varepsilon}$,

$$
\left\|\mathbf{P}_{\varepsilon}^{(1)} u_{\varepsilon}\right\|_{H^{1}\left(\Omega_{1}\right)}+\left\|\mathbf{P}_{\varepsilon}^{(2)} u_{\varepsilon}\right\|_{H^{1}\left(\Omega_{2}\right)} \leq C_{2}\left(\left\|\mathbf{F}_{\varepsilon}\right\|_{L^{2}\left(\Omega_{\varepsilon}\right)}+\left\|f_{\varepsilon}\right\|_{L^{2}\left(\Omega_{\varepsilon}\right)}\right) \leq C_{3} .
$$


Proof. The first step in the proof is to show that scattering of the values of the solution $u_{\varepsilon}$ on neighboring thin rods is small in some sense.

Here we assume for simplicity that $\gamma \equiv$ const. In general case similarly as in the proof of Theorem 4.1 [4], we should multiply the differential equation of problem (2) by a smooth cutoff function $\chi_{0}$ such that $\chi_{0}\left(x_{2}\right)=0$ for $x_{2} \geq \gamma_{0}$, and $\chi_{0}\left(x_{2}\right)=1$ for $x_{2} \leq \gamma_{0} / 2$, where $\gamma_{0}=$ $=\min _{x_{1} \in[0, a]} \gamma\left(x_{1}\right)$; and consider the function $v_{\varepsilon}=\chi_{0} u_{\varepsilon}$ which is a solution to the corresponding boundary-value problem in a thick two-level junction whose junction's body is the rectangle $[0, a] \times\left[0, \gamma_{0}\right]$.

Thus, the problem (2) is invariant under the $\varepsilon$-shift along the axis $x_{1}$. This means that the function

$$
\mathbf{U}_{\varepsilon}(x)=\varepsilon^{-1}\left(u_{\varepsilon}\left(x+\varepsilon \bar{e}_{1}\right)-u_{\varepsilon}(x)\right), \quad \bar{e}_{1}=(1,0),
$$

is a solution, $a$-periodic in $x_{1}$, to the following problem:

$$
\begin{aligned}
-\Delta_{x} \mathbf{U}_{\varepsilon}(x) & =\mathbf{F}_{\varepsilon}(x), & & x \in \Omega_{\varepsilon}, \\
\partial_{\nu} \mathbf{U}_{\varepsilon}(x) & =-\varepsilon k_{1} \mathbf{U}_{\varepsilon}(x), & & x \in \Upsilon^{(1)}(\varepsilon), \\
\partial_{\nu} \mathbf{U}_{\varepsilon}(x) & =-\varepsilon k_{2} \mathbf{U}_{\varepsilon}(x), & & x \in \Upsilon^{(2)}(\varepsilon), \\
\partial_{x_{1}}^{p} \mathbf{U}_{\varepsilon}\left(0, x_{2}\right) & =\partial_{x_{1}}^{p} \mathbf{U}_{\varepsilon}\left(a, x_{2}\right), & & x_{2} \in[0, \gamma(0)], \quad p=0,1, \\
\partial_{\nu} \mathbf{U}_{\varepsilon}(x) & =0, & & x \in \Gamma_{\varepsilon} .
\end{aligned}
$$

By virtue of Lemma 1 and condition (18), we get the following estimate:

$$
\left\|\mathbf{U}_{\varepsilon}\right\|_{H^{1}\left(\Omega_{\varepsilon}\right)} \leq C_{2}\left\|\mathbf{F}_{\varepsilon}\right\|_{L^{2}\left(\Omega_{\varepsilon}\right)} \leq C_{3}
$$

At first we extend the solution $u_{\varepsilon}$ the domain $\Omega_{1}$ by using the "linear matching"

$$
\widehat{P}_{\varepsilon}^{(1)}\left(u_{\varepsilon}\right)(x)= \begin{cases}u_{\varepsilon}, & x \in G^{(1)}(\varepsilon), \\ B_{j}^{\varepsilon}\left(x_{2}\right)+S_{j}^{\varepsilon}\left(x_{2}\right)\left(x_{1}-\varepsilon\left(j+b_{1}+\frac{h_{1}\left(x_{2}\right)}{2}\right)\right), & x \in \widetilde{Q}_{j}^{(1)}(\varepsilon),\end{cases}
$$

in the domain $\Omega_{0} \cup G^{(1)}(\varepsilon) \cup \widetilde{Q}^{(1)}(\varepsilon)$. Here

$$
\begin{gathered}
B_{j}^{\varepsilon}\left(x_{2}\right)=u_{\varepsilon}\left(\varepsilon\left(j+b_{1}+\frac{h_{1}\left(x_{2}\right)}{2}\right), x_{2}\right), \\
S_{j}^{\varepsilon}\left(x_{2}\right)=\frac{1}{\varepsilon\left(1-h_{1}\left(x_{2}\right)\right)}\left(u_{\varepsilon}\left(\varepsilon\left(j+1+b_{1}-\frac{h_{1}\left(x_{2}\right)}{2}\right), x_{2}\right)-B_{j}^{\varepsilon}\left(x_{2}\right)\right), \\
\widetilde{Q}^{(1)}(\varepsilon)=\bigcup_{j=-1}^{N} \widetilde{Q}_{j}^{(1)}(\varepsilon),
\end{gathered}
$$

ISSN 1562-3076. Нелінійні коливання, 2004, m. 7, № 3 
and the domain

$$
\widetilde{Q}_{j}^{(1)}(\varepsilon)=\left\{x: x_{2} \in\left(-d_{1},-\varepsilon\right), x_{1} \in\left(\varepsilon\left(j+b_{1}+\frac{h_{1}\left(x_{2}\right)}{2}\right), \varepsilon\left(j+1+b_{1}-\frac{h_{1}\left(x_{2}\right)}{2}\right)\right)\right\}
$$

is situated between two rods $G_{j}^{(1)}(\varepsilon)$ and $G_{j+1}^{(1)}(\varepsilon)$. In the case of the extreme rods, we perform the $a$-periodic extension of problem (2) with respect to the axis $O x_{1}$.

Without the loss of generality, we can assume here that $h_{1}$ is smooth on $\left[-d_{1}, 0\right]$. It is easy to calculate that

$$
\begin{aligned}
\left\|\widehat{P}_{\varepsilon}^{(1)}\left(u_{\varepsilon}\right)\right\|_{H^{1}\left(\widetilde{Q}_{j}^{(1)}(\varepsilon)\right)}^{2} & \\
= & \int_{\widetilde{Q}_{j}^{(1)}(\varepsilon)}\left|B_{j}^{\varepsilon}\left(x_{2}\right)+S_{j}^{\varepsilon}\left(x_{2}\right)\left[x_{1}-\varepsilon\left(j+b_{1}+\frac{h_{1}\left(x_{2}\right)}{2}\right)\right]\right|^{2} d x+ \\
& +\int_{\widetilde{Q}_{j}^{(1)}(\varepsilon)} \mid\left(B_{j}^{\varepsilon}\left(x_{2}\right)\right)^{\prime}+S_{j}^{\varepsilon}\left(x_{2}\right)+\left(S_{j}^{\varepsilon}\left(x_{2}\right)\right)^{\prime} \times \\
& \times\left.\left[x_{1}-\varepsilon\left(j+b_{1}+\frac{h_{1}\left(x_{2}\right)}{2}\right)\right]\right|^{2} d x .
\end{aligned}
$$

Further, we will not indicate that functions $B_{j}^{\varepsilon}, S_{j}^{\varepsilon}$ and $h_{1}$ are depending on $x_{2}$ if it doesn't lead to a confusion. By using the inequality $(a+b)^{2} \leq 2 a^{2}+2 b^{2}$ and properties (1) for $h_{1}$, we get

$$
\begin{array}{rl}
\left\|\widehat{P}_{\varepsilon}^{(1)}\left(u_{\varepsilon}\right)\right\|_{H^{1}\left(\widetilde{Q}_{j}^{(1)}(\varepsilon)\right)}^{2} & 2 \int_{\widetilde{Q}_{j}^{(1)}(\varepsilon)}\left(B_{j}^{\varepsilon}\right)^{2}+ \\
& +2 \int_{\widetilde{Q}_{j}^{(1)}(\varepsilon)}\left(S_{j}^{\varepsilon}\right)^{2}\left[x_{1}-\varepsilon\left(j+b_{1}+\frac{h_{1}}{2}\right)\right]^{2} d x+ \\
& +2 \int_{\widetilde{Q}_{j}^{(1)}(\varepsilon)}\left(\left(B_{j}^{\varepsilon}\right)^{\prime}\right)^{2} d x+4 \int_{\widetilde{Q}_{j}^{(1)}(\varepsilon)}\left(S_{j}^{\varepsilon}\right)^{2} d x+ \\
& +4 \int_{\widetilde{Q}_{j}^{(1)}(\varepsilon)}\left(\left(S_{j}^{\varepsilon}\right)^{\prime}\right)^{2}\left[x_{1}-\varepsilon\left(j+b_{1}+\frac{h_{1}}{2}\right)\right]^{2} d x .
\end{array}
$$


Now, taking into account the geometry of the domain $\widetilde{Q}_{j}^{(1)}(\varepsilon)$, we deduce

$$
\begin{aligned}
& \left\|\widehat{P}_{\varepsilon}^{(1)}\left(u_{\varepsilon}\right)\right\|_{H^{1}\left(\widetilde{Q}_{j}^{(1)}(\varepsilon)\right)}^{2} \leq 4 \varepsilon\left(1-m_{0}\right) \int_{-d_{1}}^{-\varepsilon}\left[\left(B_{j}^{\varepsilon}\right)^{2}+\left(\left(B_{j}^{\varepsilon}\right)^{\prime}\right)^{2}+\left(S_{j}^{\varepsilon}\right)^{2}\right] d x_{2}+4 \int_{-d_{1}}^{-\varepsilon}\left[\left(S_{j}^{\varepsilon}\right)^{2}+\right. \\
& +\left(\left(S_{j}^{\varepsilon}\right)^{\prime}\right)^{2} \int_{\varepsilon\left(j+b_{1}+\frac{h_{1}}{2}\right)}^{\varepsilon\left(j+1+b_{1}-\frac{h_{1}}{2}\right)}\left[x_{1}-\varepsilon\left(j+b_{1}+\frac{h_{1}}{2}\right)\right]^{2} d x_{1} d x_{2} \leq \\
& \leq 4 \varepsilon\left(1-m_{0}\right) \int_{-d_{1}}^{-\varepsilon}\left[\left(B_{j}^{\varepsilon}\right)^{2}+\left(\left(B_{j}^{\varepsilon}\right)^{\prime}\right)^{2}+\left(S_{j}^{\varepsilon}\right)^{2}\right] d x_{2}+ \\
& +4 \frac{\varepsilon^{3}\left(1-m_{0}\right)^{3}}{3} \int_{-d_{1}}^{-\varepsilon}\left[\left(S_{j}^{\varepsilon}\right)^{2}+\left(\left(S_{j}^{\varepsilon}\right)^{\prime}\right)^{2}\right] d x_{2} \leq \\
& \leq C_{1}\left\{\varepsilon \int_{-d_{1}}^{-\varepsilon}\left[\left(B_{j}^{\varepsilon}\right)^{2}+\left(\left(B_{j}^{\varepsilon}\right)^{\prime}\right)^{2}+\left(S_{j}^{\varepsilon}\right)^{2}\right] d x_{2}+\right. \\
& \left.+\varepsilon^{3} \int_{-d_{1}}^{-\varepsilon}\left[\left(S_{j}^{\varepsilon}\right)^{2}+\left(\left(S_{j}^{\varepsilon}\right)^{\prime}\right)^{2}\right] d x_{2}\right\}
\end{aligned}
$$

Now, let us estimate each term in the right-hand side of (25) by using the following two inequalities:

$$
\begin{gathered}
u^{2}(0) \leq 2 \varepsilon^{-1} \int_{0}^{\varepsilon} u^{2}(t) d t+2 \varepsilon \int_{0}^{\varepsilon}\left(u^{\prime}(t)\right)^{2} d t \\
(u(0)-u(\varepsilon))^{2} \leq \varepsilon \int_{0}^{\varepsilon}\left(u^{\prime}\right)^{2}(t) d t
\end{gathered}
$$

that hold for every $u \in H^{1}([0, \varepsilon])$. 
By adapting (26) to our case, we obtain

$$
u_{\varepsilon}^{2}\left(\varepsilon\left(j+b_{1}+\frac{h_{1}}{2}\right), x_{2}\right) \leq 2 \varepsilon^{-1} \int_{\varepsilon\left(j+b_{1}-\frac{h_{1}}{2}\right)}^{\varepsilon\left(j+b_{1}+\frac{h_{1}}{2}\right)} u_{\varepsilon}^{2}(x) d x_{1},+2 \varepsilon \int_{\varepsilon\left(j+b_{1}-\frac{h_{1}}{2}\right)}^{\varepsilon\left(j+b_{1}+\frac{h_{1}}{2}\right)}\left(\partial_{x_{1}} u_{\varepsilon}(x)\right)^{2} d x_{1},
$$

and integrating over $\left(-d_{1},-\varepsilon\right)$, we have

$$
\varepsilon \int_{-d_{1}}^{-\varepsilon}\left(B_{j}^{\varepsilon}\right)^{2} d x_{2} \leq 2\left\{\left\|u_{\varepsilon}\right\|_{L^{2}\left(\widetilde{G}_{j}^{(1)}(\varepsilon)\right)}^{2}+\varepsilon^{2}\left\|\partial_{x_{1}} u_{\varepsilon}\right\|_{L^{2}\left(\widetilde{G}_{j}^{(1)}(\varepsilon)\right)}^{2}\right\} \leq c_{1}\left\|u_{\varepsilon}\right\|_{H^{1}\left(G_{j}^{(1)}(\varepsilon)\right)}^{2},
$$

where $\widetilde{G}_{j}^{(1)}(\varepsilon)=G_{j}^{(1)}(\varepsilon) \cap\left\{x:-d_{1}<x_{2}<-\varepsilon\right\}$.

Moreover,

$$
\begin{aligned}
& \int_{-d_{1}}^{-\varepsilon}\left(S_{j}^{\varepsilon}\right)^{2} d x_{2}= \\
& =\int_{-d_{1}}^{-\varepsilon}\left\{\frac{1}{\varepsilon\left(1-h_{1}\right)}\left[u_{\varepsilon}\left(\varepsilon\left(j+1+b_{1}-\frac{h_{1}}{2}\right), x_{2}\right)-u_{\varepsilon}\left(\varepsilon\left(j+b_{1}+\frac{h_{1}}{2}\right), x_{2}\right)\right]\right\}^{2} d x_{2} \leq \\
& \leq c_{2} \varepsilon^{-2} \int_{-d_{1}}^{-\varepsilon}\left[u_{\varepsilon}\left(\varepsilon\left(j+1+b_{1}-\frac{h_{1}}{2}\right), x_{2}\right)-u_{\varepsilon}\left(\varepsilon\left(j+b_{1}-\frac{h_{1}}{2}\right), x_{2}\right)+\right. \\
& \left.+u_{\varepsilon}\left(\varepsilon\left(j+b_{1}-\frac{h_{1}}{2}\right), x_{2}\right)-u_{\varepsilon}\left(\varepsilon\left(j+b_{1}+\frac{h_{1}}{2}\right), x_{2}\right)\right]^{2} d x_{2}= \\
& =c_{2} \varepsilon^{-2} \int_{-d_{1}}^{-\varepsilon}\left[\varepsilon \mathbf{U}_{\varepsilon}\left(\varepsilon\left(j+b_{1}-\frac{h_{1}}{2}\right), x_{2}\right)+\right. \\
& \left.+u_{\varepsilon}\left(\varepsilon\left(j+b_{1}-\frac{h_{1}}{2}\right), x_{2}\right)-u_{\varepsilon}\left(\varepsilon\left(j+b_{1}+\frac{h_{1}}{2}\right), x_{2}\right)\right]^{2} d x_{2} \leq \\
& \leq 2 c_{2} \int_{-d_{1}}^{-\varepsilon}\left[\mathbf{U}_{\varepsilon}\left(\varepsilon\left(j+b_{1}-\frac{h_{1}}{2}\right), x_{2}\right)\right]^{2} d x_{2}+ \\
& +2 c_{2} \varepsilon^{-2} \int_{-d_{1}}^{-\varepsilon}\left[u_{\varepsilon}\left(\varepsilon\left(j+b_{1}+\frac{h_{1}}{2}\right), x_{2}\right)-u_{\varepsilon}\left(\varepsilon\left(j+b_{1}-\frac{h_{1}}{2}\right), x_{2}\right)\right]^{2} d x_{2} .
\end{aligned}
$$


By (26) and (27), we have

$$
\begin{aligned}
& \int_{-d_{1}}^{-\varepsilon}\left(S_{j}^{\varepsilon}\right)^{2} d x_{2} \leq 4 c_{2} \varepsilon^{-1}\left\|\mathbf{U}_{\varepsilon}\right\|_{L^{2}\left(\widetilde{G}_{j}^{(1)}(\varepsilon)\right)}^{2}+4 c_{2} \varepsilon\left\|\partial_{x_{1}} \mathbf{U}_{\varepsilon}\right\|_{L^{2}\left(\widetilde{G}_{j}^{(1)}(\varepsilon)\right)}^{2}+ \\
& \quad+2 c_{2} \varepsilon^{-1} M_{0} \int_{-d_{1}}^{-\varepsilon} \int_{\varepsilon\left(j+b_{1}-\frac{h_{1}}{2}\right)}^{\varepsilon\left(j+b_{1}+\frac{h_{1}}{2}\right)}\left(\partial_{x_{1}} u_{\varepsilon}(x)\right)^{2} d x_{1} d x_{2} \leq \\
& \leq c_{4}\left(\varepsilon^{-1}\left\|\mathbf{U}_{\varepsilon}\right\|_{L^{2}\left(\widetilde{G}_{j}^{(1)}(\varepsilon)\right)}^{2}+\varepsilon\left\|\partial_{x_{1}} \mathbf{U}_{\varepsilon}\right\|_{L^{2}\left(\widetilde{G}_{j}^{(1)}(\varepsilon)\right)}^{2}+\varepsilon^{-1}\left\|\partial_{x_{1}} u_{\varepsilon}\right\|_{L^{2}\left(\widetilde{G}_{j}^{(1)}(\varepsilon)\right)}^{2}\right) .
\end{aligned}
$$

Thus

$$
\begin{aligned}
\varepsilon^{3} \int_{-d_{1}}^{-\varepsilon}\left(S_{j}^{\varepsilon}\right)^{2} d x_{2} & \leq \varepsilon \int_{-d_{1}}^{-\varepsilon}\left(S_{j}^{\varepsilon}\right)^{2} d x_{2} \leq \\
& \leq c_{4}\left(\left\|\mathbf{U}_{\varepsilon}\right\|_{L^{2}\left(\widetilde{G}_{j}^{(1)}(\varepsilon)\right)}^{2}+\varepsilon^{2}\left\|\partial_{x_{1}} \mathbf{U}_{\varepsilon}\right\|_{L^{2}\left(\widetilde{G}_{j}^{(1)}(\varepsilon)\right)}^{2}+\left\|\partial_{x_{1}} u_{\varepsilon}\right\|_{L^{2}\left(\widetilde{G}_{j}^{(1)}(\varepsilon)\right)}^{2}\right) \leq \\
& \leq c_{5}\left(\left\|\mathbf{U}_{\varepsilon}\right\|_{H^{1}\left(G_{j}^{(1)}(\varepsilon)\right)}^{2}+\left\|u_{\varepsilon}\right\|_{H^{1}\left(G_{j}^{(1)}(\varepsilon)\right)}^{2}\right) .
\end{aligned}
$$

Now we are going to estimate the other terms in (25),

$$
\begin{aligned}
& \int_{-d_{1}}^{-\varepsilon}\left(\left(B_{j}^{\varepsilon}\left(x_{2}\right)\right)^{\prime}\right)^{2} d x_{2}= \\
& \quad=\int_{-d_{1}}^{-\varepsilon}\left[\varepsilon \frac{h_{1}^{\prime}\left(x_{2}\right)}{2} \partial_{x_{1}} u_{\varepsilon}\left(\varepsilon\left(j+b_{1}+\frac{h_{1}}{2}\right), x_{2}\right)+\partial_{x_{2}} u_{\varepsilon}\left(\varepsilon\left(j+b_{1}+\frac{h_{1}}{2}\right), x_{2}\right)\right]^{2} d x_{2} \leq \\
& \leq \frac{M_{0}^{2}}{2} \varepsilon^{2} \int_{-d_{1}}^{-\varepsilon}\left[\partial_{x_{1}} u_{\varepsilon}\left(\varepsilon\left(j+b_{1}+\frac{h_{1}}{2}\right), x_{2}\right)\right]^{2} d x_{2}+ \\
& \quad+2 \int_{-d_{1}}^{-\varepsilon}\left(\partial_{x_{2}} u_{\varepsilon}\left(\varepsilon\left(j+b_{1}+\frac{h_{1}}{2}\right), x_{2}\right)\right)^{2} d x_{2} .
\end{aligned}
$$


If we apply to the last two integrals in (31) the same calculation of (28) we obtain

$$
\begin{gathered}
\varepsilon \int_{-d_{1}}^{-\varepsilon}\left(\left(B_{j}^{\varepsilon}\left(x_{2}\right)\right)^{\prime}\right)^{2} d x_{2} \leq c_{7}\left(\varepsilon^{2}\left\|\partial_{x_{1}} u_{\varepsilon}\right\|_{L^{2}\left(\widetilde{G}_{j}^{(1)}(\varepsilon)\right)}^{2}+\varepsilon^{4}\left\|\partial_{x_{1}}^{2} u_{\varepsilon}\right\|_{L^{2}\left(\widetilde{G}_{j}^{(1)}(\varepsilon)\right)}^{2}+\right. \\
\left.\quad+\left\|\partial_{x_{2}} u_{\varepsilon}\right\|_{L^{2}\left(\widetilde{G}_{j}^{(1)}(\varepsilon)\right)}^{2}+\varepsilon^{2}\left\|\partial_{x_{1}, x_{2}}^{2} u_{\varepsilon}\right\|_{L^{2}\left(\widetilde{G}_{j}^{(1)}(\varepsilon)\right)}^{2}\right) \leq \\
\leq c_{7}\left(\left\|\nabla u_{\varepsilon}\right\|_{L^{2}\left(\widetilde{G}_{j}^{(1)}(\varepsilon)\right)}^{2}+\varepsilon^{2}\left\|u_{\varepsilon}\right\|_{H^{2}\left(\widetilde{G}_{j}^{(1)}(\varepsilon)\right)}^{2}\right) .
\end{gathered}
$$

To estimate the norm $\left\|u_{\varepsilon}\right\|_{H^{2}\left(\widetilde{G}_{j}^{(1)}(\varepsilon)\right)}^{2}$, we use the second energy inequality [5] with the smooth cut-off function

$$
\chi_{\varepsilon}\left(x_{2}\right)=\left\{\begin{array}{l}
0, \quad x_{2} \geq-\frac{\varepsilon}{2} \\
1, \quad x_{2} \leq-\varepsilon
\end{array}\right.
$$

We obtain that $\varepsilon^{2}\left\|u_{\varepsilon}\right\|_{H^{2}\left(\widetilde{G}_{j}^{(1)}(\varepsilon)\right)}^{2} \leq c\left(\left\|u_{\varepsilon}\right\|_{H^{1}\left(G_{j}^{(1)}(\varepsilon)\right)}^{2}+\left\|f_{\varepsilon}\right\|_{L^{2}\left(G_{j}^{(1)}(\varepsilon)\right)}^{2}\right)$. So,

$$
\varepsilon \int_{-d_{1}}^{-\varepsilon}\left(\left(B_{j}^{\varepsilon}\left(x_{2}\right)\right)^{\prime}\right)^{2} d x_{2} \leq c_{8}\left(\left\|u_{\varepsilon}\right\|_{H^{1}\left(G_{j}^{(1)}(\varepsilon)\right)}^{2}+\left\|f_{\varepsilon}\right\|_{L^{2}\left(G_{j}^{(1)}(\varepsilon)\right)}^{2}\right) .
$$

Now, let us estimate the integral of square of the derivative,

$$
\begin{aligned}
\left(S_{j}^{\varepsilon}\left(x_{2}\right)\right)^{\prime}= & \frac{h_{1}^{\prime}\left(x_{2}\right)}{1-h_{1}\left(x_{2}\right)} S_{j}^{\varepsilon}\left(x_{2}\right)+\frac{1}{\varepsilon\left(1-h_{1}\left(x_{2}\right)\right)}\left\{\varepsilon \partial_{x_{2}} \mathbf{U}_{\varepsilon}\left(\varepsilon\left(j+b_{1}-\frac{h_{1}}{2}\right), x_{2}\right)+\right. \\
& \left.+\left[\partial_{x_{2}} u_{\varepsilon}\left(\varepsilon\left(j+b_{1}-\frac{h_{1}}{2}\right), x_{2}\right)-\partial_{x_{2}} u_{\varepsilon}\left(\varepsilon\left(j+b_{1}+\frac{h_{1}}{2}\right), x_{2}\right)\right]\right\}- \\
& -\frac{h_{1}^{\prime}\left(x_{2}\right)}{2\left(1-h_{1}\left(x_{2}\right)\right)}\left[\partial_{x_{1}} u_{\varepsilon}\left(\varepsilon\left(j+1+b_{1}-\frac{h_{1}}{2}\right), x_{2}\right)+\partial_{x_{1}} u_{\varepsilon}\left(\varepsilon\left(j+b_{1}+\frac{h_{1}}{2}\right), x_{2}\right)\right] .
\end{aligned}
$$

Taking into account the properties of $h_{1}$, its derivative (see (1)), estimate (29) and applying (26) 
and (27), we deduce

$$
\begin{aligned}
\int_{-d_{1}}^{-\varepsilon}\left(\left(S_{j}^{\varepsilon}\right)^{\prime}\right)^{2} d x_{2} \leq & \\
\leq & c_{9}\left[c_{4}\left(\varepsilon^{-1}\left\|\mathbf{U}_{\varepsilon}\right\|_{L^{2}}^{2}\left(\widetilde{G}_{j}^{(1)}(\varepsilon)\right)+\varepsilon\left\|\partial_{x_{1}} \mathbf{U}_{\varepsilon}\right\|_{L^{2}\left(\widetilde{G}_{j}^{(1)}(\varepsilon)\right)}^{2}+\varepsilon^{-1}\left\|\partial_{x_{1}} u_{\varepsilon}\right\|_{L^{2}\left(\widetilde{G}_{j}^{(1)}(\varepsilon)\right)}^{2}\right)+\right. \\
& +c_{10}\left(\varepsilon^{-1}\left\|\partial_{x_{2}} \mathbf{U}_{\varepsilon}\right\|_{L^{2}\left(\widetilde{G}_{j}^{(1)}(\varepsilon)\right)}^{2}+\varepsilon\left\|\partial_{x_{1}, x_{2}}^{2} \mathbf{U}_{\varepsilon}\right\|_{L^{2}\left(\widetilde{G}_{j}^{(1)}(\varepsilon)\right)}^{2}\right)+ \\
& +c_{11} \varepsilon^{-1}\left\|\partial_{x_{1}, x_{2}}^{2} u_{\varepsilon}\right\|_{L^{2}\left(\widetilde{G}_{j}^{(1)}(\varepsilon)\right)}^{2}+c_{12}\left(\varepsilon^{-1}\left\|\partial_{x_{1}} u_{\varepsilon}\right\|_{L^{2}\left(\widetilde{G}_{j+1}^{(1)}(\varepsilon)\right)}^{2}+\varepsilon\left\|\partial_{x_{1}}^{2} u_{\varepsilon}\right\|_{L^{2}\left(\widetilde{G}_{j+1}^{(1)}(\varepsilon)\right)}^{2}\right)+ \\
& \left.+c_{13}\left(\varepsilon^{-1}\left\|\partial_{x_{1}} u_{\varepsilon}\right\|_{L^{2}\left(\widetilde{G}_{j}^{(1)}(\varepsilon)\right)}^{2}+\varepsilon\left\|\partial_{x_{1}}^{2} u_{\varepsilon}\right\|_{L^{2}\left(\widetilde{G}_{j}^{(1)}(\varepsilon)\right)}^{2}\right)\right] \leq \\
\leq & c_{14}\left(\varepsilon^{-1}\left\|\mathbf{U}_{\varepsilon}\right\|_{H^{1}\left(\widetilde{G}_{j}^{(1)}(\varepsilon)\right)}^{2}+\varepsilon\left\|\nabla \mathbf{U}_{\varepsilon}\right\|_{L^{2}\left(\widetilde{G}_{j}^{(1)}(\varepsilon)\right)}^{2}+\varepsilon\left\|\mathbf{U}_{\varepsilon}\right\|_{H^{2}\left(\widetilde{G}_{j}^{(1)}(\varepsilon)\right)}^{2}\right. \\
& \left.+\varepsilon^{-1}\left\|u_{\varepsilon}\right\|_{H^{2}\left(\widetilde{G}_{j}^{(1)}(\varepsilon) \cup \widetilde{G}_{j+1}^{(1)}(\varepsilon)\right)}^{2}+\varepsilon^{-1}\left\|\nabla u_{\varepsilon}\right\|_{L^{2}\left(\widetilde{G}_{j}^{(1)}(\varepsilon) \cup \widetilde{G}_{j+1}^{(1)}(\varepsilon)\right)}^{2}\right)
\end{aligned}
$$

Again applying the second energy inequality as above, we have

$$
\begin{gathered}
\varepsilon^{3} \int_{-d_{1}}^{-\varepsilon}\left(\left(S_{j}^{\varepsilon}\right)^{\prime}\right)^{2} \leq c_{15}\left(\varepsilon^{2}\left\|\mathbf{U}_{\varepsilon}\right\|_{H^{1}\left(G_{j}^{(1)}(\varepsilon)\right)}^{2}+\varepsilon^{4}\left\|\mathbf{U}_{\varepsilon}\right\|_{H^{1}\left(\widetilde{G}_{j}^{(1)}(\varepsilon)\right)}^{2}+\varepsilon^{2}\left\|\mathbf{F}_{\varepsilon}\right\|_{L^{2}\left(G_{j}^{(1)}(\varepsilon)\right)}^{2}+\right. \\
\left.+\left\|u_{\varepsilon}\right\|_{H^{1}\left(G_{j}^{(1)}(\varepsilon) \cup G_{j+1}^{(1)}(\varepsilon)\right)}^{2}+\left\|f_{\varepsilon}\right\|_{L^{2}\left(G_{j}^{(1)}(\varepsilon) \cup G_{j+1}^{(1)}(\varepsilon)\right)}^{2}\right) .
\end{gathered}
$$

Thus, by (28), (30), (32) and (33), the right-hand side of (25) is estimated in the following way:

$$
\begin{aligned}
\left\|\widehat{P}_{\varepsilon}^{(1)}\left(u_{\varepsilon}\right)\right\|_{H^{1}\left(\widetilde{Q}_{j}^{(1)}(\varepsilon)\right)}^{2} & \leq c_{16}\left(\left\|u_{\varepsilon}\right\|_{H^{1}\left(G_{j}^{(1)}(\varepsilon)\right)}^{2}+\left\|\mathbf{U}_{\varepsilon}\right\|_{H^{1}\left(G_{j}^{(1)}(\varepsilon)\right)}^{2}+\left\|f_{\varepsilon}\right\|_{L^{2}\left(G_{j}^{(1)}(\varepsilon)\right)}^{2}+\right. \\
& \left.+\varepsilon^{2}\left\|\mathbf{F}_{\varepsilon}\right\|_{L^{2}\left(G_{j}^{(1)}(\varepsilon)\right)}^{2}+\left\|u_{\varepsilon}\right\|_{H^{1}\left(G_{j}^{(1)}(\varepsilon) \cup G_{j+1}^{(1)}(\varepsilon)\right)}^{2}+\left\|f_{\varepsilon}\right\|_{L^{2}\left(G_{j}^{(1)}(\varepsilon) \cup G_{j+1}^{(1)}(\varepsilon)\right)}^{2}\right) .
\end{aligned}
$$

Summing (33) over $j$ from -1 to $N$, using Lemma 1 and (3), we get

$$
\left\|\widehat{P}_{\varepsilon}^{(1)}\left(u_{\varepsilon}\right)\right\|_{H^{1}\left(G^{(1)}(\varepsilon) \cup \widetilde{Q}^{(1)}(\varepsilon)\right)}^{2} \leq c_{17}\left(\left\|f_{\varepsilon}\right\|_{L^{2}\left(\Omega_{\varepsilon}\right)}^{2}+\left\|\mathbf{F}_{\varepsilon}\right\|_{L_{2}\left(\Omega_{\varepsilon}\right)}^{2}\right) .
$$


Now it remains to extend $\widehat{P}_{\varepsilon}^{(1)}\left(u_{\varepsilon}\right)$ to

$$
\begin{gathered}
T_{j}^{(1)}(\varepsilon)=\left\{x: x_{2} \in(-\varepsilon, 0), x_{1} \in\left(\varepsilon\left(j+b_{1}+2^{-1} h_{1}\left(x_{2}\right)\right), \varepsilon\left(j+1+b_{1}-2^{-1} h_{1}\left(x_{2}\right)\right)\right)\right\}, \\
j=-1,0,1, \ldots, N .
\end{gathered}
$$

Since the domains $T_{j}^{(1)}(\varepsilon), j=-1,0,1, . ., N$, are equal (each of this domain can be obtained from $T_{0}^{(1)}(\varepsilon)$ by a parallel shift along the axis $O x_{1}$ ), we use results about extension operators in perforated domains [6]. It follows from these results that there exist an extension operator $\mathfrak{P}_{\varepsilon}^{(1)}: H^{1}\left(G^{(1)}(\varepsilon) \cup \widetilde{Q}^{(1)}(\varepsilon)\right) \mapsto H^{1}\left(\Omega_{1}\right)$, uniformly bounded in $\varepsilon$.

Thus, the extension operator $\mathbf{P}_{\varepsilon}^{(1)}:=\mathfrak{P}_{\varepsilon}^{(1)} \circ \widehat{P}_{\varepsilon}^{(1)}$ is constructed and it satisfies the uniform estimate (19). Similarly we can construct the operator $\mathbf{P}_{\varepsilon}^{(2)}: H^{1}\left(\Omega_{0} \cup G^{(2)}(\varepsilon)\right) \mapsto H^{1}\left(\Omega_{2}\right)$ which also satisfies (19).

The theorem is proved.

4. Convergence theorem. To prove this theorem we should pass to the limit in the integral identity (4) as $\varepsilon \rightarrow 0$. For this we will use identity (9), the extension operators constructed in Section 3 , and the following characteristic functions

$$
\chi_{\varepsilon}^{(i)}(x):=\chi^{(i)}\left(\frac{x_{1}}{\varepsilon}, x_{2}\right)=\left\{\begin{array}{ll}
0, & x \in \Omega_{0}, \\
1, & x \in G^{(i)}(\varepsilon), \\
0, & x \in D_{i} \backslash G^{(i)}(\varepsilon),
\end{array} \quad i=1,2 .\right.
$$

We can assume that these functions are $\varepsilon$-periodic with respect to $x_{1}$. Similarly as in Section 4 [1], we can prove that $\chi_{\varepsilon}^{(i)} \rightarrow h_{i}$ weakly in $L_{2}\left(D_{i}\right)$ as $\varepsilon \rightarrow 0$.

Theorem 2. Let $u_{\varepsilon}$ be a weak solution to problem (2). Then

$$
\left.\left(u_{\varepsilon}\right)\right|_{\Omega_{0}} \rightarrow v_{0}^{+},\left.\quad\left(\mathbf{P}_{\varepsilon}^{(1)} u_{\varepsilon}\right)\right|_{D_{1}} \rightarrow v_{0}^{1,-},\left.\quad\left(\mathbf{P}_{\varepsilon}^{(2)} u_{\varepsilon}\right)\right|_{D_{2}} \rightarrow v_{0}^{2,-}
$$

weakly in $H^{1}\left(\Omega_{0}\right), H^{1}\left(D_{1}\right), H^{1}\left(D_{2}\right)$ respectively as $\varepsilon \rightarrow 0$, where the vector-valued function

$$
\mathbf{v}_{0}(x)=\left\{\begin{array}{cc}
v_{0}^{+}(x), & x \in \Omega_{0} \\
v_{0}^{1,-}(x), & x \in D_{1} \\
v_{0}^{2,-}(x), & x \in D_{2}
\end{array}\right.
$$


is the unique weak solution to the following problem:

$$
\begin{array}{rlrl}
-\Delta_{x} v_{0}^{+}(x) & =f_{0}(x), & & x \in \Omega_{0}, \\
\partial_{x_{1}}^{p} v_{0}^{+}\left(0, x_{2}\right) & =\partial_{x_{1}}^{p} v_{0}^{+}\left(a, x_{2}\right), \quad p=0,1, & x_{2} \in[0, \gamma(0)], \\
\partial_{\nu} v_{0}^{+}(x) & =0, & & x \in \Gamma_{\gamma}, \\
-\partial_{x_{2}}\left(h_{1}\left(x_{2}\right) \partial_{x_{2}} v_{0}^{1,-}(x)\right)+2 k_{1} v_{0}^{1,-}(x) & =h_{1}\left(x_{2}\right) f_{0}(x), & & x \in D_{1}, \\
\partial_{x_{2}} v_{0}^{1,-}\left(x_{1},-d_{1}\right) & =0, & & x_{1} \in I_{0}, \\
-\partial_{x_{2}}\left(h_{2}\left(x_{2}\right) \partial_{x_{2}} v_{0}^{2,-}(x)\right)+2 k_{2} v_{0}^{2,-}(x) & =h_{2}\left(x_{2}\right) f_{0}(x), & & x \in D_{2}, \\
\partial_{x_{2}} v_{0}^{2,-}\left(x_{1},-d_{2}\right) & =0, & & x_{1} \in I_{0}, \\
v_{0}^{+}\left(x_{1}, 0\right)=v_{0}^{1,-}\left(x_{1}, 0\right) & =v_{0}^{2,-}\left(x_{1}, 0\right), & & x_{1} \in I_{0}, \\
\partial_{x_{2}} v_{0}^{+}\left(x_{1}, 0\right) & =h_{1}(0) \partial_{x_{2}} v_{0}^{1,-}\left(x_{1}, 0\right)+ & & \\
& +h_{2}(0) \partial_{x_{2}} v_{0}^{2,-}\left(x_{1}, 0\right), & & x_{1} \in I_{0},
\end{array}
$$

where $\Gamma_{\gamma}=\left\{x: x_{2}=\gamma\left(x_{1}\right), x_{1} \in I_{0}\right\}$.

Proof. With the help of (9), the extension operators $\mathbf{P}_{\varepsilon}^{(i)}$ and the functions $\chi_{\varepsilon}^{(i)}, i=1,2$, we rewrite identity (4) in the following way:

$$
\begin{aligned}
\int_{\Omega_{0}} \nabla u_{\varepsilon} \cdot \nabla \varphi d x & +\sum_{i=1}^{2}(\int_{D_{i}} \chi_{\varepsilon}^{(i)}(x) \nabla\left(\mathbf{P}_{\varepsilon}^{(i)} u_{\varepsilon}\right) \cdot \nabla(\varphi) d x+\underbrace{\left.\varepsilon k_{i} \int_{0}^{a}\left(\chi_{\varepsilon}^{(i)} \mathbf{P}_{\varepsilon}^{(i)} u_{\varepsilon} \varphi\right)\right|_{x_{2}=-d_{i}}}_{A_{1}} d x_{1}+ \\
& \left.+2 k_{i} \int_{D_{i}} \frac{\sqrt{1+\varepsilon^{2} 4^{-1}\left|h_{i}^{\prime}\left(x_{2}\right)\right|^{2}}}{h_{i}\left(x_{2}\right)} \chi_{\varepsilon}^{(i)}(x)\left(\mathbf{P}_{\varepsilon}^{(i)} u_{\varepsilon}\right)(x) \varphi(x) d x\right)= \\
& =2 \sum_{i=1}^{2} \sum_{G^{(i)}(\varepsilon)} k_{i}\left(\frac{x_{1}}{\varepsilon}\right) \frac{\sqrt{1+\varepsilon^{2} 4^{-1}\left|h_{i}^{\prime}\left(x_{2}\right)\right|^{2}}}{h_{i}\left(x_{2}\right)} \partial_{x_{1}}\left(u_{\varepsilon} \varphi\right) d x \\
& +\int_{\Omega_{0}} f_{\varepsilon}(x) \varphi(x) d x+\sum_{i=1}^{2} \int_{D_{i}} \chi_{\varepsilon}^{(i)}(x) f_{\varepsilon}(x) \varphi(x) d x \quad \forall \varphi \in H_{\|, x_{1}}^{1}\left(\Omega_{2}\right),
\end{aligned}
$$

where $H_{\Perp, x_{1}}^{1}\left(\Omega_{2}\right)=\left\{\varphi \in H^{1}\left(\Omega_{2}\right): \varphi\left(0, x_{2}\right)=\varphi\left(a, x_{2}\right)\right.$ for $\left.x_{2} \in(0, \gamma(0))\right\}$.

Because of (3), (5), (19), the sequences

$$
\left\{\chi_{\varepsilon}^{(i)} \partial_{x_{j}}\left(\mathbf{P}_{\varepsilon}^{(i)} u_{\varepsilon}\right)\right\}_{\varepsilon>0}, \quad j=1,2
$$


are bounded in $L_{2}\left(D_{i}\right), i=1,2$. Therefore, we can choose a subsequence of $\{\varepsilon\}$ ( still denoted by $\{\varepsilon\})$ such that $\chi_{\varepsilon}^{(i)} \partial_{x_{j}}\left(\mathbf{P}_{\varepsilon}^{(i)} u_{\varepsilon}\right) \rightarrow \sigma_{j}^{(i)}$ weakly in $L_{2}\left(D_{i}\right), j=1,2, i=1,2$, and

$$
\left.\left(u_{\varepsilon}\right)\right|_{\Omega_{0}} \rightarrow v_{0}^{+},\left.\quad\left(\mathbf{P}_{\varepsilon}^{(1)} u_{\varepsilon}\right)\right|_{D_{1}} \rightarrow v_{0}^{1,-},\left.\quad\left(\mathbf{P}_{\varepsilon}^{(2)} u_{\varepsilon}\right)\right|_{D_{2}} \rightarrow v_{0}^{2,-}
$$

weakly in $H^{1}\left(\Omega_{0}\right), H^{1}\left(D_{1}\right), H^{1}\left(D_{2}\right)$ and strongly in $L^{2}\left(\Omega_{0}\right), L^{2}\left(D_{1}\right), L^{2}\left(D_{2}\right)$ respectively as $\varepsilon \rightarrow 0$. Since $\left.\left(u_{\varepsilon}\right)\right|_{I_{0}}=\left.\left(\mathbf{P}_{\varepsilon}^{(1)} u_{\varepsilon}\right)\right|_{I_{0}}=\left.\left(\mathbf{P}_{\varepsilon}^{(2)} u_{\varepsilon}\right)\right|_{I_{0}}$, the traces of limit functions are equal as well, i.e., $v_{0}^{+}\left(x_{1}, 0\right)=v_{0}^{1,-}\left(x_{1}, 0\right)=v_{0}^{2,-}\left(x_{1}, 0\right), x_{1} \in I_{0}$.

Obviously, the summands $A_{1}$ and $A_{2}$ in (37) vanishe as $\varepsilon \rightarrow 0$. Now, passing to the limit in (37) and taking (3), (38) into account, we obtain

$$
\begin{aligned}
\int_{\Omega_{0}} \nabla v_{0}^{+} \cdot \nabla \varphi d x+ & \sum_{i=1}^{2}\left(\int_{D_{i}} \sum_{j=1}^{2} \sigma_{j}^{(i)}(x) \partial_{x_{j}} \varphi(x) d x+2 k_{i} \int_{D_{i}} v_{0}^{i,-} \varphi d x\right)= \\
& =\int_{\Omega_{0}} f_{0}(x) \varphi(x) d x+\sum_{i=1}^{2} \int_{D_{i}} h_{i}\left(x_{2}\right) f_{0}(x) \varphi(x) d x, \quad \varphi \in H_{\|, x_{1}}^{1}\left(\Omega_{2}\right) .
\end{aligned}
$$

Next we should find $\sigma_{j}^{(i)}, j=1,2, i=1,2$.

In order to determine $\sigma_{1}^{(i)}, i=1,2$, we consider the integral identity (4) with the following test functions :

$\psi_{1}(x)=\varepsilon\left\{\begin{array}{ll}0, & x \in \Omega_{0}, \\ Y\left(x_{1} / \varepsilon\right) \phi_{1}(x), & x \in G^{(1)}(\varepsilon), \\ 0, & x \in G^{(2)}(\varepsilon),\end{array} \quad \psi_{2}(x)=\varepsilon \begin{cases}0, & x \in \Omega_{0}, \\ 0, & x \in G^{(1)}(\varepsilon), \\ Y\left(x_{1} / \varepsilon\right) \phi_{2}(x), & x \in G^{(2)}(\varepsilon),\end{cases}\right.$

where $\phi_{1}$ and $\phi_{2}$ are arbitrary functions from $C_{0}^{\infty}\left(D_{1}\right)$ and $C_{0}^{\infty}\left(D_{2}\right)$ respectively. It is obvious that $\psi_{1}$ and $\psi_{2}$ belong to $H_{\|, x_{1}}^{1}\left(\Omega_{\varepsilon}\right)$. As a result, we get

$$
\int_{D_{1}} \chi_{\varepsilon}^{(1)}(x) \partial_{x_{1}} \mathbf{P}_{\varepsilon}^{(1)}\left(u_{\varepsilon}\right) \phi_{1} d x=\mathcal{O}(\varepsilon), \quad \int_{D_{2}} \chi_{\varepsilon}^{(2)}(x) \partial_{x_{1}} \mathbf{P}_{\varepsilon}^{(2)}\left(u_{\varepsilon}\right) \phi_{2} d x=\mathcal{O}(\varepsilon), \quad \varepsilon \rightarrow 0
$$

whence $\sigma_{1}^{(1)} \equiv 0$ and $\sigma_{1}^{(2)} \equiv 0$.

Next let us define $\sigma_{2}^{(1)}$. Take any function $\phi \in C_{0}^{\infty}\left(D_{1}\right)$ and perform the following calculati- 
ons:

$$
\begin{aligned}
\int_{D_{1}} \chi_{\varepsilon}^{(1)}(x) \partial_{x_{2}}\left(\mathbf{P}_{\varepsilon}^{(1)} u_{\varepsilon}(x)\right) \phi(x) d x & =\sum_{j=0}^{N-1} \int_{G_{j}^{(1)}(\varepsilon)} \partial_{x_{2}}\left(u_{\varepsilon}(x)\right) \phi(x) d x= \\
& =\sum_{j=0}^{N-1}\left(\int_{\Upsilon_{j}^{(1, \pm)}(\varepsilon)} u_{\varepsilon} \phi \alpha_{2}^{(1)}\left(x_{2}, \varepsilon\right) d l_{x}-\int_{G_{j}^{(1)}(\varepsilon)} u_{\varepsilon} \partial_{x_{1}} \phi d x\right)= \\
& =-\left.2^{-1} \int_{-d_{1}}^{0} h_{1}^{\prime}\left(x_{2}\right) \sum_{j=0}^{N-1}\left(u_{\varepsilon} \phi\right)\right|_{x_{1}=\varepsilon\left(j+b_{1} \pm h_{1}\left(x_{2}\right) / 2\right)} d x_{2}- \\
& -\int_{D_{1}} \chi_{\varepsilon}^{(1)}(x)\left(\mathbf{P}_{\varepsilon}^{(1)} u_{\varepsilon}\right) \partial_{x_{2}} \phi d x=: B_{1}(\varepsilon)+B_{2}(\varepsilon) .
\end{aligned}
$$

Here $\alpha_{2}^{(1)}\left(x_{2}, \varepsilon\right)=-\varepsilon h_{1}^{\prime}\left(x_{2}\right)\left(2 \sqrt{1+\varepsilon^{2} 4^{-1}\left(h_{1}^{\prime}\left(x_{2}\right)\right)^{2}}\right)^{-1}$ is the second coordinate of the outward unit normal $\nu_{ \pm}^{(1)}$ (see (8) to the lateral surfaces $\Upsilon_{j}^{(1, \pm)}(\varepsilon)$ of the thin $\operatorname{rod} G_{j}^{(1)}(\varepsilon)$. It is easy to verify that

$$
\lim _{\varepsilon \rightarrow 0} B_{2}(\varepsilon)=-\int_{D_{1}} h_{1}\left(x_{2}\right) v_{0}^{1,-}(x) \partial_{x_{2}} \phi(x) d x .
$$

To find the limit of $B_{1}(\varepsilon)$ we rewrite this value in the following way:

$$
\begin{aligned}
B_{1}(\varepsilon)=-2^{-1} \varepsilon \int_{-d_{1}}^{0} h_{1}^{\prime}\left(x_{2}\right)\left(\sum_{j=0}^{N-1} \int_{\varepsilon\left(j+b_{1}-h_{0}\left(x_{2}\right) / 2\right)}^{\varepsilon\left(j+b_{1}+h_{0}\left(x_{2}\right) / 2\right)} \partial_{x_{1}}\left(u_{\varepsilon} \phi\right) d x_{1}\right) d x_{2}- \\
-\varepsilon \int_{-d_{1}}^{0} h_{1}^{\prime}\left(x_{2}\right)\left(\left.\sum_{j=0}^{N-1}\left(\left(u_{\varepsilon}-v_{0}^{1,-}\right) \phi\right)\right|_{x_{1}=\varepsilon\left(j+b_{1}-h_{0}\left(x_{2}\right) / 2\right)}\right) d x_{2}- \\
\quad-\int_{-d_{1}}^{0} h_{1}^{\prime}\left(x_{2}\right)\left(\left.\sum_{j=0}^{N-1}\left(v_{0}^{1,-} \phi\right)\right|_{x_{1}=\varepsilon\left(j+b_{1}-h_{0}\left(x_{2}\right) / 2\right)}(\varepsilon(j+1)-\varepsilon j)\right) d x_{2} .
\end{aligned}
$$

The first term in (42) is bounded by $\varepsilon\left\|u_{\varepsilon}\right\|_{H^{1}\left(G^{(1)}(\varepsilon)\right)}\|\phi\|_{H^{1}\left(D_{1}\right)}$. Due to estimate (26), the second term in (42) is estimated by the value

$$
c_{1}\left(\left\|\mathbf{P}_{\varepsilon}^{(1)} u_{\varepsilon}-v_{0}^{1,-}\right\|_{L^{2}\left(G^{(1)}(\varepsilon)\right)}+\varepsilon^{2}\left\|\partial_{x_{1}}\left(\mathbf{P}_{\varepsilon}^{(1)} u_{\varepsilon}-v_{0}^{1,-}\right)\right\|_{L^{2}\left(G^{(1)}(\varepsilon)\right)}\right)\|\phi\|_{H^{1}\left(D_{1}\right)} .
$$


Since for almost all points $x_{2} \in\left(-d_{1}, 0\right)$ the function $v_{0}^{1,-} \in H^{1}(0, a)$, the inner sum in the third term in (42) is the Riemann sum for the integral $\int_{0}^{a} v_{0}^{1,-} \phi d x_{1}$. Then in view of Lebesgue's Theorem and Fubini's Theorem, the limit of the third term is equal to

$$
-\int_{D_{1}} h_{1}^{\prime}\left(x_{2}\right) v_{0}^{1,-}(x) \phi(x) d x
$$

Passing to the limit in (40) and taking (41) - (44) into account, we get

$$
\sigma_{2}^{(1)}(x)=h_{1}\left(x_{2}\right) \partial_{x_{2}} v_{0}^{1,-}(x), \quad x \in D_{1} .
$$

Similarly we deduce that $\sigma_{2}^{(2)}(x)=h_{2}\left(x_{2}\right) \partial_{x_{2}} v_{0}^{2,-}(x), \quad x \in D_{2}$.

Thus, we obtain that the vector-valued function $\mathbf{v}_{0}$ satisfies the following identity:

$$
\begin{aligned}
\int_{\Omega_{0}} \nabla_{x} v_{0}^{+} \cdot \nabla_{x} \varphi d x+ & \sum_{i=1}^{2} \int_{D_{i}}\left(h_{i}\left(x_{2}\right) \partial_{x_{2}} v_{0}^{i,-}(x) \partial_{x_{2}} \varphi(x)+2 k_{i} v_{0}^{i,-}(x) \varphi(x)\right) d x= \\
& =\int_{\Omega_{0}} f_{0}(x) \varphi(x) d x+\sum_{i=1}^{2} \int_{D_{i}} h_{i}\left(x_{2}\right) f_{0}(x) \varphi(x) d x \quad \forall \varphi \in H_{\|, x_{1}}^{1}\left(\Omega_{2}\right) .
\end{aligned}
$$

Identity (45) is the corresponding integral identity for problem (36) in the following anizotropic Sobolev vector-space:

$$
\begin{gathered}
\mathcal{H}_{0}=\left\{\mathbf{u}=\left(u_{0}, u_{1}, u_{2}\right) \in \mathcal{V}_{0}:=L^{2}\left(\Omega_{0}\right) \times L^{2}\left(D_{1}\right) \times L^{2}\left(D_{2}\right) \mid\right. \\
u_{0} \in H^{1}\left(\Omega_{0}\right), \quad u_{0}\left(0, x_{2}\right)=u_{0}\left(a, x_{2}\right) \text { for } x_{2} \in(0, \gamma(0)) ; \\
\exists \partial_{x_{2}} u_{1} \in L^{2}\left(D_{1}\right) ; \quad \exists \partial_{x_{2}} u_{2} \in L^{2}\left(D_{2}\right) ; \\
\left.u_{0}\left(x_{1}, 0\right)=u_{1}\left(x_{1}, 0\right)=u_{2}\left(x_{1}, 0\right), x_{1} \in I_{0}\right\}
\end{gathered}
$$

with the scalar product

$$
(\mathbf{u}, \mathbf{v})_{\mathcal{H}_{0}}=\int_{\Omega_{0}} \nabla u_{0} \cdot \nabla v_{0} d x+\sum_{i=1}^{2} \int_{D_{i}}\left(h_{i}\left(x_{2}\right) \partial_{x_{2}} u_{i} \partial_{x_{2}} v_{i}+2 k_{i} u_{i} v_{i}\right) d x
$$

Obviously, the space $\mathcal{H}_{0}$ continuously embeds in $\mathcal{V}_{0}$.

By using standard Hilbert space methods, we can state that there exists a unique weak solution $v_{0} \in \mathcal{H}$ to problem (36), which is called the limit problem for problem (2). It should be noted that in the rectangles $D_{1}$ and $D_{2}$ we have ordinary differential equations with respect to $x_{2}$ and there are no boundary conditions on the vertical sides of $D_{i}, i=1,2$. 
Due to the uniqueness of the solution to problem (36), the above reasoning holds for any subsequence of $\{\varepsilon\}$ chosen at the beginning of the proof. Therefore, the theorem is proved.

Example. In the case $h_{1} \equiv$ const and $h_{2} \equiv$ const, we can reduce problem (36) to some boundary-value problem in the junction's body. By solving these ordinary differential equations with regard to the Neumann conditions and the first transmission condition in $I_{0}$, we find

$$
\begin{aligned}
v_{0}^{i,-}(x)=- & \frac{1}{\rho_{i}} \int_{-d_{i}}^{x_{2}} \sinh \left(\rho_{i}\left(x_{2}-t\right)\right) f_{0}\left(x_{1}, t\right) d t+ \\
& +\frac{\cosh \left(\rho_{i}\left(x_{2}+d_{i}\right)\right)}{\cosh \left(\rho_{i} d_{i}\right)}\left(v_{0}^{+}\left(x_{1}, 0\right)-\frac{1}{\rho_{i}} \int_{-d_{i}}^{0} \sinh \left(\rho_{i} t\right) f_{0}\left(x_{1}, t\right) d t\right), \quad x \in D_{i},
\end{aligned}
$$

where $\rho_{i}=\sqrt{2 k_{i} h_{i}^{-1}}, \quad i=1,2$. Putting these functions in the second transmission condition, we obtain the following problem:

$$
\begin{aligned}
-\Delta_{x} v_{0}^{+}(x) & =f_{0}(x), & & x \in \Omega_{0}, \\
\partial_{x_{1}}^{p} v_{0}^{+}\left(0, x_{2}\right) & =\partial_{x_{1}}^{p} v_{0}^{+}\left(a, x_{2}\right), p=0,1, & & x_{2} \in[0, \gamma(0)], \\
\partial_{\nu} v_{0}^{+}(x) & =0, & & x \in \Gamma_{\gamma}, \\
\partial_{x_{2}} v_{0}^{+}\left(x_{1}, 0\right) & =\left(\sum_{i=1}^{2} h_{i} \rho_{i} \tanh \left(\rho_{i} d_{i}\right)\right) v_{0}^{+}\left(x_{1}, 0\right),+\widehat{f}_{0}\left(x_{1}\right), & & x_{1} \in I_{0},
\end{aligned}
$$

where

$$
\widehat{f}_{0}\left(x_{1}\right)=-\sum_{i=1}^{2} h_{i} \int_{-d_{i}}^{0}\left(\cosh \left(\rho_{i} t\right)+\tanh \left(\rho_{i} d_{i}\right) \sinh \left(\rho_{i} t\right)\right) f_{0}\left(x_{1}, t\right) d t .
$$

Problem (47) is a classical boundary-value problem with the Robin condition on $I_{0}$. Obviously, it has a unique weak solution from $H^{1}\left(\Omega_{0}\right)$.

1. De Maio U., Mel'nyk T. A. Homogenization of the Robin problem in a thick multi-structures of type 3:2:2 // Asymptotic Analysis (to appear).

2. Mel'nyk T. A., Nazarov S. A. Asymptotics of the Neumann spectral problem solution in a domain of "thick comb"type // Trudy Seminara imeni I.G. Petrovskogo. - 1996. - 19. - P. 138-173 (in Russian), and English transl.: J. Math. Sci. - 1997. - 85, № 6. - P. 2326-2346.

3. Mel'nyk T. A. Homogenization of the Poisson equation in a thick periodic junction // Z. Anal. und ihre Anwend. - 1999. - 18, № 4. - P. 953-975.

4. Mel'nyk T. A. Eigenmodes and pseudo-eigenmodes of thick multi-level junctions // Proc. Int. Conf. "Days on Diffraction-2004" (St. Petersburg, June 29-July 2, 2004). - P. 51-52.

5. Ladyzhenskaya O. A. The boundary value problems of mathematical physics. - Berlin: Springer, 1985. $295 \mathrm{p}$.

6. Oleinik O. A., Yosifian G. A., and Shamaev A. S. Mathematical problems in elasticity and homogenization. - Amsterdam: North-Holland, 1992. - 310 p. 